\title{
Simulation of Field Enhancement Effects in Carbon Nanotubes
}

\author{
R. C. Smith", J. D. Carey and S. R. P. Silva \\ Advanced Technology Institute, School of Electronics and Physical Sciences, \\ University of Surrey, Guildford, GU2 7XH, United Kingdom
}

\begin{abstract}
Simulations of electric field enhancement factor, $\beta$, for isolated metallic carbon nanotubes (CNT) are reported. We show that $\beta$ is dependent not only on the geometry of the tubes, but also on the location of the anode electrode. We also highlight the effect of field screening due to boundary conditions of the simulation package. Finally, we give an expression for $\beta$ as a function of CNT height, radius, and anode to cathode separation.
\end{abstract}

\section{Introduction}

Since the identification of carbon nanotubes (CNT) [1] there has been considerable interest in their use in vacuum microelectronics and nanoelectronics [2]. This has been brought about by sustained research in the field of carbon based cold cathodes coupled with the ability to alter the geometric field enhancement factor, $\beta$, by tailoring the physical dimensions (length and radius) of the tubes. The role of $\beta$ is the enhancement of the applied macroscopic field such that under the action of the local electric field efficient tunnelling of electrons can occur from the Fermi level through a potential barrier.

\section{Simulation}

The CNT were simulated, using the Silvaco ${ }^{\text {тм }}$ package, in two dimensions having height $h$, radius $r$, vertically aligned with a hemispherical cap on a grounded substrate separated by a distance D from a flat anode in a workarea of width $\mathrm{W}$ as shown in Fig. 1. In this configuration the CNT and substrate represent an equipotential surface.

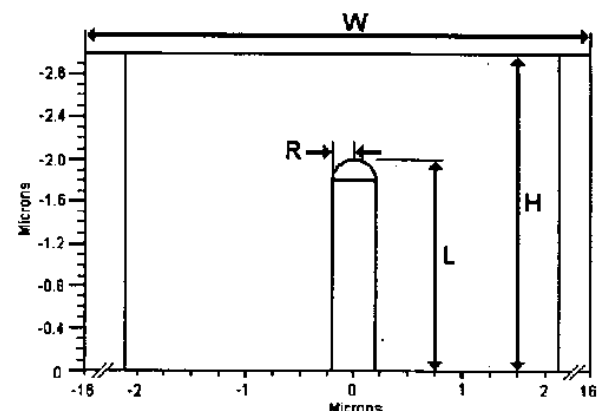

Fig. 1. Layout of simulation.

\section{Results and discussion}

The simulation package has inbuilt boundary conditions which mirrors to the left and right of the workarea. Whilst this is important for device calculations, it means that when simulating a CNT in the centre of the workarea as shown in Fig. 1, there are mirrored CNTs to each side, screening the electric field. We show that these effects become less important when the workarea width is greater than twice the height of the CNT. We investigate the role of the anode to cathode separation, D, on enhancement factors, which is calculated by local field divided by applied field on both CNT and hemispheres. A first test of a hemisphere with D=6, 20 and 36 $\mu \mathrm{m}$ show a low threshold field at $\mathrm{D}=6 \mu \mathrm{m}$, but, higher and constant values for $\mathrm{D}=20$ and $36 \mu \mathrm{m}$. Fig. 2. shows the variation of enhancement factor which saturates at high $\mathrm{D}$, but increases at low $\mathrm{D}$ for hemispheres of radius 1,2 , and $4 \mu \mathrm{m}$. It can be seen that the saturated value of enhancement factor is 2 in all three cases, which we can tie in by solving a Laplace equation in two dimensions for a hemisphere.

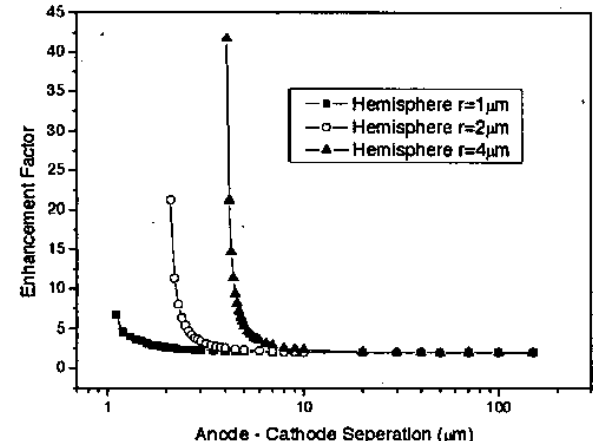

0-7803-8397ig/94/\$20i

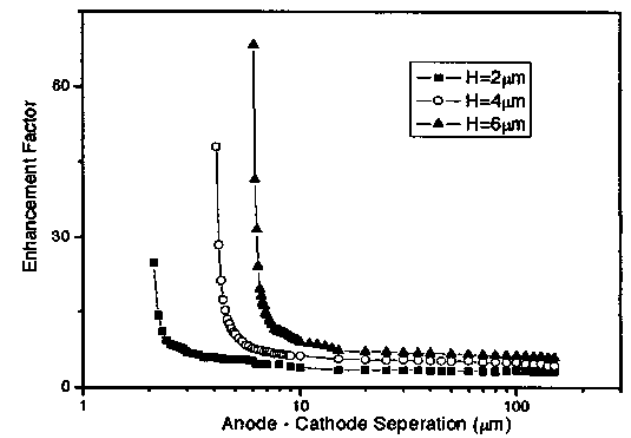

176

Fig. 3. CNT enhancement factor 
Fig. 3. shows the enhancement factors for three CNTs of radius $200 \mathrm{~nm}$ and heights 2,4 , and $6 \mu \mathrm{m}$. A similar trend of that of Fig. 2. can be seen. However in this case the saturated values of enhancement factors are different for each $\mathrm{CNT}$ suggesting a relationship for $\beta$ is based on just $h$ and $r$. The saturated region in all simulated hemispheres and CNTs becomes apparent when D is greater than three times the CNT height.

The widely used and accepted approximation that $\beta=h / r$ cis shown not to be applicable in al cases. CNTs of aspect ratios $(\mathrm{h} / \mathrm{r})$ between 1 and 500 were simulated and the enhancement factor at high D was extracted to ensure no effects from the anode plane were observed. When $\log \beta$ was plotted against $\log h / r$ a straight-line relationship was found. For the expression of $\beta=h / r$ to be true, this line had to have a gradient of 1 . However in this case the gradient was 0.38 . We further went on to show that in the high D case the expression for $\beta$, when $\alpha=2$, is:

$$
\beta=(1+\sqrt{h / \alpha r})^{0.5}
$$

In order to derive an expression for estimating the geometric enhancement factor for CNTs of given height and radius, in a vacuum separated by anode and cathode electrode distance D apart, and correctly simulated so any effects of screening due to simulation boundary conditions are at a minimum, there are two distinct halves to consider. Firstly, as seen in Figs. 2 and 3, the role of $D$ on $\beta$ is high at low D, and negligible at high D. For the second half of the expression the values of the converged enhancement factors are a factor of CNT geometry alone and not D, as shown in equation 1 . We conclude that the following expression is only valid for low $\mathrm{D}$ and is given by:

$$
\frac{D}{D-h}
$$

The equation above tends to 1 at high $\mathrm{D}$, however, it is important that upon analysing the recorded data for enhancement factors, the converged lines do not have a gradient of 0 . They decrease slightly as $\mathrm{D}$ increases. We can therefore say that equations 1 and 2 can be added together to obtain a realistic approximation for enhancement factors for all values of $h, r$ and $D$, given by:

$$
\beta=\frac{D}{D-h}+\left(1+\sqrt{\frac{h}{\alpha r}}\right)
$$

\section{Conclusions}

We have shown in this study the importance in understanding the simulation sof tware when attempting to simulate isolated CNTs. The boundary conditions of the simulator mirror the workarea, which can cause problems associated with adjacent tubes screening the field and reducing the emission current.

In examining the enhancement factor of first hemispheres and then CNTs we have shown that the anode to cathode separation plays an important role and affects $\beta$ greatly at low values of $D$. At high $D$ we give an empirical relationship for $\beta$, based on the CNT $h$ and $r$ alone and is not dependant on D.

\section{Acknowledgements}

The authors would like to thank the EPSRC Portfolio Partnership and Carbon Based Electronics programmes for funding this research.

\section{References}

1 S. Iijima, Nature. 56, 354 (1991).

2 A. G. Rinzler, J. H. Hafner, P. Nikolaev, L. Lou, S.G. Kim, D. Tománek, P. Nordlander, D.T. Colbert, and R.E. Smalley, Science. 269, 1550 (1995) 